\author{
E. Schaden \\ P. G. Metnitz \\ G. Pfanner \\ S. Heil \\ T. Pernerstorfer \\ P. Perger \\ H. Schoechl \\ D. Fries \\ M. Guetl \\ S. Kozek-Langenecker
}

\title{
Coagulation Day 2010: an Austrian survey on the routine of thromboprophylaxis in intensive care
}

Received: 31 August 2011

Accepted: 3 March 2012

Published online: 24 March 2012

(C) Copyright jointly held by Springer and ESICM 2012

Electronic supplementary material

The online version of this article (doi:10.1007/s00134-012-2533-0) contains supplementary material, which is available to authorized users.

E. Schaden $(\bowtie) \cdot$ P. G. Metnitz

Department of Anesthesiology, General

Intensive Care and Pain Control, Medical

University of Vienna, Waehringer Guertel 18-20, 1090 Vienna, Austria

e-mail: eva.schaden@meduniwien.ac.at

Tel.: +43-1-404004102

Fax: +43-1-404004028

\section{G. Pfanner}

Department of Anesthesiology and Intensive Care, Landeskrankenhaus Feldkirch, Feldkirch, Austria

\section{S. Heil}

Department of Anaesthesia and Intensive Care, Wilhelminenspital, Vienna, Austria

T. Pernerstorfer

Department of Anesthesiology and Intensive Care, Konventhospital

Barmherzige Brüder, Linz, Austria

\section{P. Perger}

Department of Anaesthesia and Intensive Care, Krankenhaus Hietzing, Vienna, Austria

H. Schoechl

Department of Anaesthesiology and Intensive Care, AUVA Trauma Hospital, Salzburg, Austria

\section{Fries}

Department of General and Surgical Critical Care Medicine, Innsbruck Medical University, Innsbruck, Austria

\section{Guetl}

Department of Anesthesiology and Intensive Care Medicine, Medical

University of Graz, Graz, Austria

\section{S. Kozek-Langenecker}

Department of Anaesthesia and Intensive Care, Evangelical Hospital Vienna, Vienna, Austria

Abstract Purpose: Venous thromboembolism (VTE) is a common but often overlooked lifethreatening complication of critical illness. The aim of this cross-sectional survey was to assess current practice of thromboprophylaxis as well as adherence to international guidelines. Methods: After ethics committee approval, all intensive care units in Austrian hospitals treating adult patients were invited to participate in this web-based survey. Anonymized data on each patient treated at the participating intensive care units on Coagulation Day 2010 were collected using an electronic case report form. Risk assessment, choice and monitoring of anticoagulants, means of mechanical prophylaxis, and demographic data were recorded. Results: Data from 325 critically ill patients were collected. Patients had a median of four risk factors for thrombosis and $6 \%$ suffered from VTE. Of the 325 patients, $80 \%$ received low molecular weight heparins subcutaneously, $10 \%$ received unfractionated heparin intravenously, $1 \%$ received alternative anticoagulants and $9 \%$ received no pharmacological prophylaxis. Mechanical prophylaxis was used in $49 \%$ with a predominant use of graduated compression stockings. In $39 \%$ a combination of pharmacological and mechanical prophylaxis was applied and $5 \%$ received no prophylaxis at all. Overall guideline adherence was $40 \%$ on Coagulation Day 2010. Conclusion: Current practice of thromboprophylaxis is predominantly based on the administration of low molecular weight heparins prescribed at rather arbitrary doses without a discernible relationship to drug monitoring, thromboembolic risk factors, vasopressor use or fluid balance. The use of mechanical prophylaxis, evaluation of risk scores and overall guideline adherence must be further encouraged by education, training and communication.

Keywords Critical illness · Prevention of thromboembolism, low molecular weight heparin - Compression stockings, intermittent pneumatic compression devices 


\section{Introduction}

Venous thromboembolism (VTE) with its clinical manifestations of deep venous thrombosis (DVT) and pulmonary embolism (PE) is common in intensive care patients [1]. Despite prophylaxis, more than $15 \%$ of critically ill patients suffer from VTE [2]. This alarming prevalence of VTE in critical illness may in part be caused by the simultaneous occurrence of multiple risk factors such as age, immobilization, sepsis, trauma and/or decreased bioavailability of subcutaneous (s.c.) low molecular weight heparins (LMWH) [3-6]. VTE is lifethreatening and DVT, even if initially asymptomatic, may provoke post-thrombotic syndrome as an irreversible lifelong consequence $[4,7]$. These clinical manifestations without doubt decrease quality of life after surviving critical illness and contribute to increased overall healthcare costs [8]. Despite their impact on patient safety and patient outcome, intensive care physicians appear to be more concerned about bleeding than thrombotic events [9].

International and interdisciplinary recommendations exist on the prevention of VTE in critical illness [2]. Management should include an individualized risk assessment and pharmacological thromboprophylaxis-if not contraindicated - using unfractionated heparin (UFH) or LMWH (level of recommendation grade 1, level of evidence A). For critical care patients who are at high risk of bleeding, the optimal use of mechanical thromboprophylaxis with graduated compression stockings (GCS) and/or intermittent pneumatic compression (IPC) at least until the bleeding risk decreases is recommended (level of recommendation grade 1 , level of evidence A). When the high bleeding risk decreases, pharmacological thromboprophylaxis should be substituted for or added to the mechanical thromboprophylaxis (level of recommendation grade 1, level of evidence C). Adherence to guidelines in general is known to be low but adherence to guidelines on thromboprophylaxis in critically ill patients nationwide is unknown. Accordingly, the aim of this cross-sectional survey in Austrian intensive care units (ICU) was to assess current practice of VTE risk assessment, methods and monitoring of thromboprophylaxis as well as the degree of guideline adherence.

\section{Methods}

The survey was endorsed as a national observational network trial by the Task Force of Perioperative Coagulation and by the Intensive Care section of the Austrian Society of Anaesthesiology, Resuscitation and Intensive Care (OEGARI). The questionnaire was created by members of the Task Force in the German Language and formatted into a personalized electronic Case Report
Form (e-CRF) by an IT expert. The study was approved by the Ethics Committee of the Medical University of Vienna and the General Hospital of Vienna (no. $828 / 2010$, date of vote 12.11.2010).

We invited all ICUs and intermediate care units in Austrian hospitals treating adult patients (independent of their primary specialty) to participate in the web-based survey. As the decision to initiate VTE prophylaxis in children is still made on a case-by-case basis, paediatric and neonatal units were excluded. First we generated a list including electronic contact details of medical directors of all Austrian ICUs. In an email we informed the medical directors about the project, and asked them for their approval and assignment of an intensive care physician on their wards as our contact person. These contact persons were informed about the purpose of the survey and were instructed how to fill in the e-CRF. Contact persons were requested to deliver anonymized data on each patient who was treated at their ICU on Coagulation Day 2010, which was 1 December 2010. The term Coagulation Day was our creation for dedicating one specific day to the topic of coagulation in Austrian ICU patients. After an announcement by email 1 week in advance, an email with the link to the personal e-CRF was sent to each contact person on Coagulation Day 2010. The deadline for data input was 8 December; 2 days before this date a gentle reminder was sent by email.

\section{Questionnaire items}

In addition to demographic data the following items were evaluated in the questionnaire:

- Anticoagulants: drugs administered for pharmacological thromboprophylaxis, dosing of anticoagulants and, if any, laboratory tests used for monitoring during pharmacological thromboprophylaxis.

- Mechanical prophylaxis: means of mechanical prophylaxis.

- Risk assessment: underlying and actual diseases, known coagulation disorders and the use of risks scores. For every patient the individual risk profile was determined, including age $>40$ years, sex, body mass index (BMI) $>30 \mathrm{~kg} / \mathrm{m}^{2}$, severity of illness by the simplified acute physiology score (SAPS) II and the sequential organ failure assessment score (SOFA), presence of heart failure, active cancer, varicose veins, fractures, surgery in the past 2 weeks, central venous line, mechanical ventilation with PEEP.

- Confounding factors: use of vasopressors and positive fluid balance were also evaluated because of their potential inhibiting effect on the bioavailability of s.c. anticoagulants $[5,6]$. The size of the respective hospital and the number of beds in the ICU as well as the medical discipline (anaesthesia, internal medicine, 
surgery, neurosurgery, neurology) were also documented.

The degree of guideline adherence was assessed by comparing actual practice with international and interdisciplinary recommendations on the prevention of VTE in critical illness [2].

\section{Statistics}

Data were analysed using the $\mathrm{R}$ software environment ( $\mathrm{R}$ version 2.13.0, 2011-04-13). Descriptive data are given as number or percentage of the total number of patients if not otherwise stated. For the purpose of readability numbers and percentages were rounded up to whole numbers. For questions with multiple possible answers, each answer was analysed separately in regard to the percentage of positive and negative responses. Due to the descriptive character of the study, no $p$ values were computed.

\section{Results}

From 186 contacted medical directors of ICUs 101 responded to the invitation to participate in this national survey. Finally, 59 contact persons completed the e-CRF, which gives a response rate of $32 \%$. Five contact persons were internists, and $91 \%$ were specialists in anaesthesia and intensive care working in ICUs with a median of eight beds (range 6-12 beds). More than half of the patients $(54 \%)$ were admitted to ICUs in hospitals with more than 600 beds.

Data from 325 critically ill patients were collected. The patients' characteristics are shown in Table 1. The most common admission diagnoses were postoperative state in $22 \%$, sepsis in $19 \%$, brain trauma or craniotomy in $15 \%$, trauma in $8 \%$, respiratory failure in $8 \%$, and cardiac disease in $6 \%$. Bleeding complications were the reason for admission in $2 \%$ of patients.

Table 2 summarizes the risk factors for VTE. Patients exhibited a median of four risk factors for thrombosis (range one to five). None of the responders reported the use of a score to assess individual patients' risk of thrombosis. On the day of the survey, only $4 \%$ of patients $(n=11)$ suffered from thrombosis and PE was diagnosed in $2 \%(n=5)$.

Figure 1 shows the use of anticoagulants and nonpharmacological thromboprophylaxis in the cohort. Of the 325 patients, $80 \%$ received LMWH and $10 \%$ received UFH. Two patients received alternative anticoagulants (argatroban and danaparoid) for thromboprophylaxis. Overall, $39 \%$ of all patients received GCS and $10 \%$ received IPC. In $39 \%$ of patients a combination of
Table 1 Characteristics of the study population

\begin{tabular}{ll}
\hline Characteristics & Value \\
\hline Patients $(n)$ & \\
Total & 325 \\
Male/female & $176 / 149$ \\
Age (years, mean \pm SD) & $64.0 \pm 18.3$ \\
BMI $^{\mathrm{a}}\left(\mathrm{kg} / \mathrm{m}^{2}\right.$, mean $\left.\pm \mathrm{SD}\right)$ & $26.8 \pm 5.9$ \\
SAPS II (median, interquartile range) & $33.3(22.0-44.0)$ \\
SOFA (median, interquartile range) & $7(4-10)$
\end{tabular}

${ }^{a}$ Reference range $18.5-25 \mathrm{~kg} / \mathrm{m}^{2}$

Table 2 Risk factors for VTE in the study population, based on $[3,4]$

\begin{tabular}{ll}
\hline Factor & $\%$ of patients \\
\hline Age $>40$ years & 88 \\
Central venous line & 76 \\
Surgery in the past 2 weeks & 58 \\
Mechanical ventilation with PEEP & 45 \\
Heart failure (NYHA stage 2-4) & 26 \\
BMI $>30 \mathrm{~kg} / \mathrm{m}^{2}$ & 23 \\
Active cancer & 20 \\
Sepsis & 19 \\
Varicose veins & 17 \\
Brain trauma/craniotomy & 15 \\
Fractures & 13 \\
\hline
\end{tabular}

pharmacological and mechanical prophylaxis was applied. In $83 \%$ of these patients, LMWH or UFH was combined with GCS, in $16 \%$ with IPC, and in $1 \%$ with GCS and IPC. Of the $9 \%$ of patients not receiving any pharmacological prophylaxis, $49 \%$ used GCS, $2 \%$ received IPC, and $1 \%$ both GCS and IPC. Thus overall $5 \%$ of patients received neither pharmacological nor mechanical thromboprophylaxis. Of these patients, $25 \%$ had brain trauma and/or craniotomy.

Of the patients diagnosed with thrombosis or PE, $19 \%$ received no thromboprophylaxis, $31 \%$ received only pharmacological prophylaxis, $44 \%$ received a combination of pharmacological prophylaxis and GCS, and $6 \%$ used GCS only. In none of the patients with a known thrombotic event was IPC applied.

The LMWH administration route was s.c., the reported doses varied considerably with no relationship to drug monitoring, risk factors, vasopressor use and/or fluid balance. UFH was administered as a continuous intravenous (i.v.) infusion, with the dose adjusted according to the aPTT results. LMWH was monitored using aPTT in $20 \%$ and using anti-Xa levels in $25 \%$ of patients on LMWH. Thirty seven percent of these had a creatinine clearance below $30 \mathrm{ml} / \mathrm{min}$. Conversely, $17 \%$ of patients with a creatinine clearance below $30 \mathrm{ml} / \mathrm{min}$ received LMWH without anti-Xa monitoring. Anti-Xa was monitored in $36 \%$ of patients with brain trauma/craniotomy who received LMWH. 

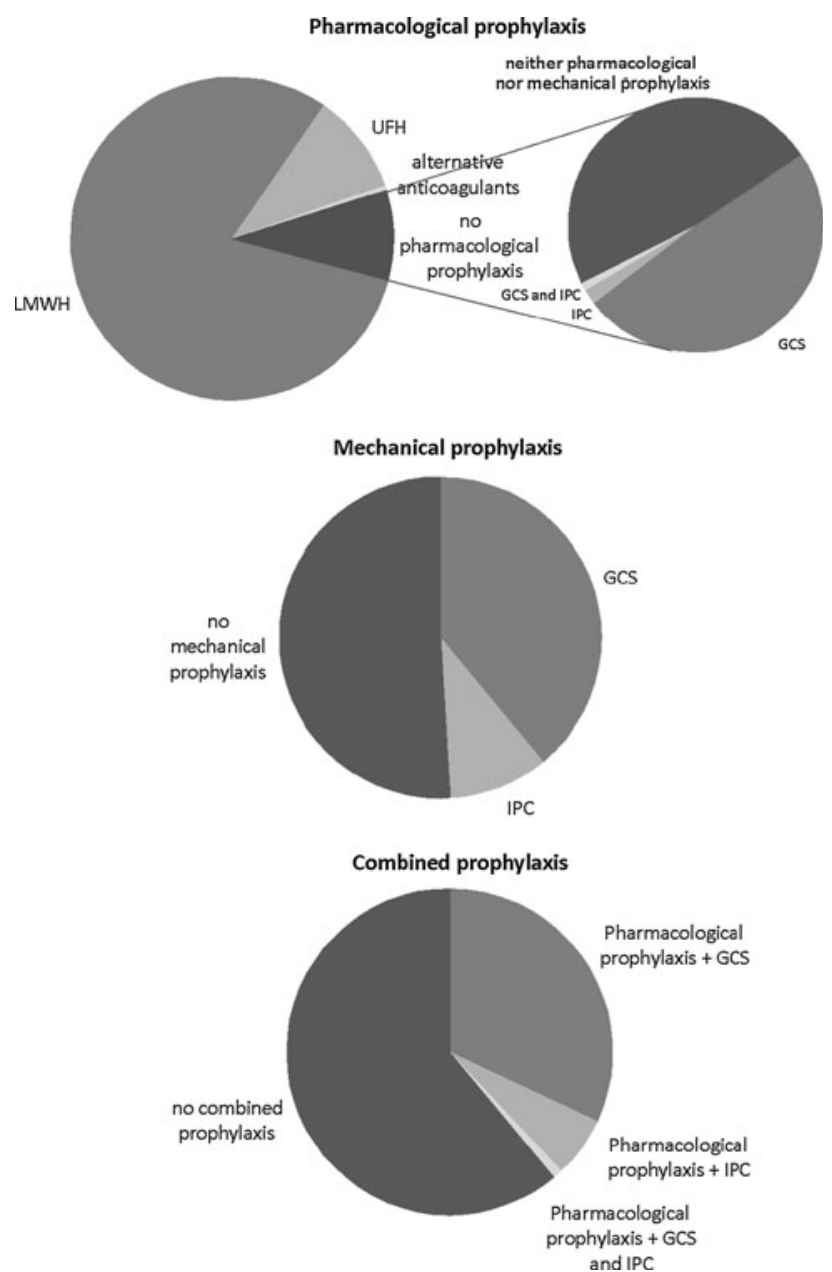

Fig. 1 Use of pharmacological and mechanical thromboprophylaxis alone or in combination ( $L M W H$ low molecular weight heparin, $U F H$ unfractionated heparin, GCS graduated compression stockings, $I P C$ intermittent pneumatic compression)

There was no relevant difference between hospitals with more or fewer than 600 beds regarding the use of pharmacological prophylaxis (LMWH $52 \%$ vs. $48 \%$, UFH $63 \%$ vs. $37 \%$ ) and GCS (44\% vs. $56 \%$ ), but $71 \%$ of patients receiving IPC were treated in hospitals with more than 600 beds. Regarding LMWH monitoring, $84 \%$ of those patients in whom anti-Xa was used were in big hospitals with more than 600 beds, while $71 \%$ of those in whom aPTT was used were in smaller hospitals with fewer than 600 beds.

\section{Discussion}

The present cross-sectional survey in Austrian ICUs addressed the current practice of VTE risk assessment as well as methods and monitoring of thromboprophylaxis. Confirming and extending previous surveys, guideline adherence was moderate $[10,11]$. A combination of pharmacological and mechanical methods for thromboprophylaxis is recommended for critically ill patients at high risk of bleeding and/or DVT [2]. Despite the presence of a median of four risk factors, the Coagulation Day 2010 survey showed the implementation of this particular recommendation in only $39 \%$ of patients. A previous survey performed in France and Canada in 2003 found that combined prophylactic modalities were used in less than $10 \%$ [10]. We can only speculate on the reasons for this attitude but it may-at least in part-be due to insufficient communication and knowledge of published guidelines, as well as limited recognition of expert panels and their consensus statements.

The most severe violation of the VTE prophylaxis guidelines was observed in $5 \%$ of patients in our survey who received neither pharmacological nor mechanical prophylaxis. Our questionnaire did not assess the reasons for therapeutic decisions, but not implementing anticoagulation could be explained by missed opportunities for prescription, combined contraindications against the use of anticoagulants and mechanical thromboprophylaxis, or the perceived risk of bleeding in these patients. In fact, guidelines do not define "safe" time-points for starting anticoagulants and these are left to the clinician's discretion. The limited use of mechanical devices was particularly pronounced in patients with brain trauma/ craniotomy. Although there is strong evidence for the use of especially IPC in neurosurgical patients (level of recommendation grade 1, level of evidence A) [2], only five of these patients in our survey received mechanical prophylaxis. Cochrane analyses have shown than that IPC is beneficial in preventing VTE in high-risk patients and have revealed an additional benefit of IPC if combined with pharmacological prophylaxis [12]. IPC can also be used easily in obese patients. In none of the patients diagnosed with a thrombotic event in our survey was IPC provided. It has to be taken into account that a recent DVT is a contraindication to the use of IPC.

Another Cochrane review has shown that GCS is beneficial in various groups of hospitalized patients [13]. Despite this evidence, only about a third of the patients in our survey received (additive) mechanical prophylaxis. The limited use may be due to educational, economic and logistic issues [14]. Nursing personnel are often concerned about patient discomfort and adverse events of GCS such as blisters and pressure sores [11, 14]. However, in the studies included in the above-mentioned Cochrane review no serious adverse events were documented [13]. In a recent study in orthopaedic surgery, comfort during IPC was rated by the patients as good (7.6 on a scale from 1 to 10) [15]. Adequate dosing of IPC has been reported to be more than $6 \mathrm{~h}$ per day, and this needs to be acknowledged [15]. Given the scientific evidence of the efficacy and patient satisfaction, more efforts are needed to increase the acceptance of mechanical 
thromboprophylaxis among health-care providers-both intensivists and nurses.

There is an ongoing debate regarding the bioavailability of LMWH administered s.c. in critically ill patients receiving vasopressors and/or high amounts of fluids [5, 6]. Robinson et al. [16] have recently suggested that the dose of s.c. enoxaparin should be increased to achieve anti-Xa activities in the recommended range [17]. In this context it needs to be born in mind that target ranges are different for different LMWHs, and that it is still unknown if targets derived from pharmacological studies in healthy volunteers are indeed applicable to the critically ill population with major, heterogeneous and unpredictable modifications in pharmacokinetics and pharmacodynamics, as well as changes in haemostatic competence. Another problem is the only weak relationship between anti-Xa activity and clinical occurrence of symptomatic and asymptomatic VTE [18-20]. However, determination of anti-Xa activity is recommended if renal elimination of LMWH is impaired [2]. Also in patients at risk of bleeding into delicate regions (e.g. the central nervous system), drug monitoring could be useful. The uncertainty of clinicians regarding the utility and practicability of the antiXa testing is reflected by its limited use in our survey (25\% of patients on LMWH), even among those critically ill patients with renal insufficiency and brain trauma/craniotomy. In $20 \%$ of patients who received LMWH, aPTT was analysed, although it is not sensitive enough for quantifying the biological effects of LMWH thromboprophylaxis. Only severe overdosing or accumulation of LMWH prolongs aPTT. The prescribed doses of LMWH varied considerably among patients, obviously as a result of lack of targeting based on the laboratory results for adequate drug monitoring, and without a clear relationship to risk factors, vasopressor use or fluid balance.

According to our survey, some clinicians in Austria try to avoid all these obstacles linked to s.c. LMWH by continuous prophylactic i.v. administration of UFH, although there is no evidence supporting this approach for primary VTE prevention. Apart from that, the usually targeted aPTT prolongation in Austria of 1.5-2.5 times the control value fulfils the criterion for therapeutic anticoagulation, and may contribute to an increased bleeding risk. Further limitations of conventional coagulation tests have to be considered. In an acute phase response with elevated factor VIII levels, aPTT may be inappropriate for monitoring the effects of UFH [21]. Some alternative anticoagulants as well as the new direct thrombin inhibitors modify the results of conventional coagulation tests such as aPTT [22]. Altogether, alternative laboratory methods may help to quantitatively assess the effects of anticoagulant drugs [23].

The risk profile of intensive care patients in our study arises from a combination of multiple risk factors. The patients displayed a median of four risk factors. A combination of risk factors is considered to be associated with an increased risk of VTE; e.g. patients heterozygous for factor V Leiden have a three- to sevenfold increased risk. Oral contraceptives confer a two- to threefold increased risk. In the presence of both risk factors, the relative risk is increased 34-fold [4]. Critically ill patients exhibit mainly temporary risk factors such as sepsis, fractures, surgery, or central venous catheterization (Table 2). The magnitude of individual risk elevation due to combinations of temporary risk factors is not clear; however, despite the respective recommendation (level of recommendation grade 1, level of evidence A) [2] individual risk scores were not determined.

Interestingly, the incidence of clinical thrombosis was very low in our patients. It is unclear whether VTE did not occur or was simply not diagnosed. Our survey data were based on clinical observations only and not on sophisticated laboratory or sonographic techniques. VTE can be clinically inapparent. In a study of 261 ICU patients, $9.6 \%$ had sonographically proven proximal DVT, and in all but one patient this was clinically unsuspected [24]. It needs to be considered that both silent and overt DVT are risk factors for the potentially life-threatening event of PE, which is also often overlooked and only found after death [4]. DVT with or without clinical symptoms can be complicated by post-thrombotic syndrome which usually develops months later-out of sight; learning from cause and effect is therefore not possible for intensivists. However, the rate of post-thrombotic syndrome is suggested to be high and may represent a chronic severe residue after surviving critical illness. It is noteworthy that post-thrombotic syndrome can be prevented by consistent use of GCS $[4,7]$.

We obtained our survey data from ICUs with a median of eight beds which is the size of an average Austrian ICU (range 6-12 beds). According to our survey, hospital size is relevant for management strategies in the ICU. Anti-Xa testing was mainly performed in big hospitals with more than 600 beds, whereas aPTT was mainly used in smaller hospitals. Almost three-quarters of patients receiving IPC were treated in big hospitals. It can be speculated that the costs and the provision of materials and qualified personnel determine the availability of mechanical prophylaxis and laboratory testing.

A limitation of our survey was that some aspects, e.g. concerning the general haemostatic management, were not included. We tried to keep the survey short and userfriendly. Furthermore, the results of the survey mirror current practice of thromboprophylaxis in 325 critically ill patients treated in 2010 in Austria, and should not be transferred directly to other settings and countries. Nevertheless, compared to previous surveys [10, 11], our results may indicate an improvement in overall adherence to VTE prophylaxis guidelines over the last decade. The response rate was higher than in Italy $(17 \%)$ but lower than in France $(45 \%)$ and Germany $(50 \%)$ [10, 11, 25]. However, we believe that opinion polls concerning guidelines in wards, departments or hospitals do not reflect reality regarding putting of these recommendations 
into practice. We hope that the Austrian ICU data bank created by the Austrian Society of Anaesthesiology will receive more input from our future network trials among all disciplines involved in intensive care therapy.

Finally, unclear questions in our e-CRF and errors in data reporting could have contributed to unexpected responses. This is a potential limitation of any surveypaper-based or web-based.

\section{Conclusion}

Inherent problems in diagnosing VTE and its complications may nourish the general underestimation of its high clinical relevance and need for adequate prevention. Increasing awareness by education, training and communication may improve quality of care and guideline adherence which was $40 \%$ in our 2010 Austrian survey. Especially the use of mechanical prophylaxis combined with anticoagulants and appropriate drug-monitoring should be encouraged.

Acknowledgments This project was funded by the Medical Scientific Fund of the Mayor of the City of Vienna. We thank the members of the Task Force on Perioperative Coagulation and of the Intensive Care section of the Austrian Society of Anesthesiology, Resuscitation and Intensive Care (OEGARI) for their support. Special thanks to J. Gassner-Uphues and O. Diesenbacher for the design of the web-based questionnaire and technical support.

\section{References}

1. Levi M (2008) Hemostasis and thrombosis in critically ill patients. Semin Thromb Hemost 34:415-416

2. Hirsh J, Guyatt G, Albers GW, Harrington R, Schünemann HJ; American College of Chest Physicians (2008) Antithrombotic and thrombolytic therapy: American College of Chest Physicians EvidenceBased Clinical Practice Guidelines (8th Edition). Chest 133(6 Suppl):110S$112 \mathrm{~S}$

3. Anderson FA Jr, Spencer FA (2003) Risk factors for venous thromboembolism. Circulation 107(23 Suppl 1):I9-16

4. Cushman M (2007) Epidemiology and risk factors for venous thrombosis. Semin Hematol 44:62-69

5. Dörffler-Melly J, de Jonge E, Pont AC, Meijers J, Vroom MB, Büller HR, Levi M (2002) Bioavailability of subcutaneous low-molecular-weight heparin to patients on vasopressors. Lancet 359:849-850

6. Rommers MK, Van der Lely N, Egberts TC, van den Bemt PM (2006) Anti-Xa activity after subcutaneous administration of dalteparin in ICU patients with and without subcutaneous oedema: a pilot study. Crit Care 10:R93

7. Kahn SR (2011) The post thrombotic syndrome. Thromb Res 127(Suppl 3): S89-S92
8. Cohen AT, Agnelli G, Anderson FA, Arcelus JI, Bergqvist D, Brecht JG, Greer IA, Heit JA, Hutchinson JL, Kakkar AK, Mottier D, Oger E, Samama MM, Spannagl M; VTE Impact Assessment Group in Europe (VITAE) (2007) Venous thromboembolism (VTE) in Europe: the number of VTE events and associated morbidity and mortality. Thromb Haemost 98:756-764

9. Crowther MA, Cook DJ (2008) Preventing venous thromboembolism in critically ill patients. Semin Thromb Hemost 34:469-474

10. Lacherade JC, Cook D, Heyland D, Chrusch C, Brochard L, Brun-Buisson $\mathrm{C}$; French and Canadian ICU Directors Groups (2003) Prevention of venous thromboembolism in critically ill medical patients: a Franco-Canadian cross-sectional study. J Crit Care $18: 228-237$

11. Imberti D, Ageno W (2005) A survey of thromboprophylaxis management in patients with major trauma. Pathophysiol Haemost Thromb 34:249-254

12. Kakkos SK, Caprini JA, Geroulakos G, Nicolaides AN, Stansby GP, Reddy DJ (2008) Combined intermittent pneumatic leg compression and pharmacological prophylaxis for prevention of venous thromboembolism in high-risk patients. Cochrane Database Syst Rev 4:CD005258

13. Sachdeva A, Dalton M, Amaragiri SV, Lees T (2010) Elastic compression stockings for prevention of deep vein thrombosis. Cochrane Database Syst Rev 7:CD001484
14. Fritz E, Them C, Hackl JM (2005) Routine nursing measures for thrombosis prevention in an university hospital. Pflege 18:43-50

15. Eisele R, Kinzl L, Koelsch T (2007) Rapid-inflation intermittent pneumatic compression for prevention of deep venous thrombosis. J Bone Joint Surg Am 89:1050-1056

16. Robinson S, Zincuk A, Strøm T, Larsen TB, Rasmussen B, Toft P (2010) Enoxaparin, effective dosage for intensive care patients: double-blinded, randomised clinical trial. Crit Care 14:R41

17. Levi M (2010) Adequate thromboprophylaxis in critically ill patients. Crit Care 14:142

18. Bara L, Leizorovicz A, Picolet H, Samama MM (1992) Correlation between anti-Xa and occurrence of thrombosis and haemorrhage in postsurgical patients treated with either Logiparin (LMWH) or unfractionated heparin. Post-surgery Logiparin Study Group. Thromb Res 65:641-650

19. Leizorovicz A, Bara L, Samama MM, Haugh MC (1993) Factor Xa inhibition: correlation between the plasma levels of anti-Xa activity and occurrence of thrombosis and haemorrhage. Haemostasis 23(Suppl 1):89-98

20. Bara L, Planes A, Samama MM (1999) Occurrence of thrombosis and haemorrhage, relationship with anti-Xa, anti-IIa activities, and D-dimer plasma levels in patients receiving a low molecular weight heparin, enoxaparin or tinzaparin, to prevent deep vein thrombosis after hip surgery. Br J Haematol 104:230-240 
21. Levine MN, Hirsh J, Gent M, Turpie AG, Cruickshank M, Weitz J, Anderson D, Johnson M (1994) A randomized trial comparing activated thromboplastin time with heparin assay in patients with acute venous thromboembolism requiring large daily doses of heparin. Arch Intern Med 154:49-56

22. Levy JH, Key NS, Azran MS (2010) Novel oral anticoagulants: implications in the perioperative setting.

Anesthesiology 113:726-745
23. Schaden E, Schober A, Hacker S, Spiss C, Chiari A, Kozek-Langenecker S (2010) Determination of enoxaparin with rotational thrombelastometry using the prothrombinase-induced clotting time reagent. Blood Coagul Fibrinolysis 21:256-261
24. Cook DJ, Crowther MA, Meade MO, Douketis J (2005) Prevalence, incidence, and risk factors for venous thromboembolism in medical-surgical intensive care unit patients. J Crit Care 20:309-313

25. Hilbert P, Teumer P, Stuttmann R (2008) Prevention of thromboembolism in German intensive care units: results of a nationwide survey. Anaesthesist $57: 242-250$ 